\title{
Benefits in Using Information Systems for the healthy growth of organizations
}

\author{
Izabel Pinheiro Andion ${ }^{1}$, Marcelo da Silva Andion ${ }^{1}$
}

\author{
${ }^{1}$ Universidade Paulista. Campus Manaus. Avenida Mário Ypiranga, No 4390. Parque 10 de novembro. Manaus-Amazonas- Brasil.
}

Email: ipandion@gmail.com, marceloandion1@gmail.com

Received: November 17th, 2016

Accepted: January $16^{\text {th }}, 2017$

Published: March 30th 2017

Copyright $@ 2016$ by authors and Institute of Technology Galileo of Amazon (ITEGAM) This work is licensed under the Creative Commons Attribution International License (CC BY 4.0).

http://creativecommons.org/licenses/by/4.0/

\begin{abstract}
Information systems are already widely used in companies, because it is through this mechanism that the manager makes the decision, defines strategies and modifies some aspect of the process that is not reaching the expected goal. The continuous search for competitiveness and productivity increase has led companies to invest more and more in Information Systems. Good quality information is essential for good decision making, an information system does not have to have essentially computers involved, just having several parts working together to generate information. This paper aims to analyze the different positions about the importance of information systems for organizations. It presents the results of a research, in the bibliographic approach, with information collected from theoretical references published in books, journals, scientific articles and others.
\end{abstract}

Keywords: Information systems; Organizational development; Growth of market share.

\begin{abstract}
Benefícios na Utilização de Sistemas de Informação para o crescimento saudável das organizações

\section{RESUMO}

Sistemas de informação já são muito utilizados nas empresas, pois é através desse mecanismo que o gestor faz a tomada de decisão, define estratégias e modifica algum aspecto do processo que não esteja atingindo o objetivo esperado. A busca contínua pela competitividade e pelo aumento de produtividade tem levado as empresas a investirem cada vez mais em Sistemas de Informação. Informações de boa qualidade são essenciais para uma boa tomada de decisão, um sistema de informação não precisa ter essencialmente computadores envolvidos, basta ter várias partes trabalhando entre si para gerar informações. Este trabalho tem o objetivo de analisar as diversas posições acerca da importância dos sistemas de informação para as organizações. Nele é apresentado o resultado de uma pesquisa, na abordagem bibliográfica, com informações coletadas a partir de referências teóricas publicadas em livros, revistas, artigos científicos e outros.
\end{abstract}

Palavras Chaves: Sistemas de Informação; Desenvolvimento organizacional; Crescimento da participação de Mercado.

\section{INTRODUÇÃO}

Muito antes do surgimento do computador, os sistemas de informação já se baseavam em técnicas de registros, onde os dados eram catalogados, organizados e arquivados com a finalidade da recuperação de informações, atribuições estas designadas e encontradas na figura do arquivista. Segundo [1]. " $O$ arquivista é um profissional de nível superior responsável pelo gerenciamento dos documentos e das informações arquivísticas e a sua formação tem como foco principal o tratamento especializado dos documentos e das informações arquivisticas registradas em qualquer tipo de suporte - produzidas e acumuladas por uma pessoa física ou jurídica, pública ou privada, no curso de suas atividades".

Aparentemente simples, este método exigia um grande esforço humano para manter os dados atualizados e organizados bem como para recuperá-los. Isso sem mencionar o grau de dificuldade para efetuar o cruzamento de dados e análises das informações. 
O advento da tecnologia propiciou que os esforços físicos fossem substituídos por softwares específicos capazes de extrair dados, transformando-os em conhecimento e estes em informações. A partir desta nova era do conhecimento, a informação começou a fluir com uma velocidade muito superior aos controles físicos, surgindo novas formas controlar, organizar, armazenar e disponibilizar informações para a tomada de decisões.

Segundo [2] um "sistema de informação é uma série de elementos ou componentes inter-relacionados que coletam (entrada), manipulam e armazenam (processo), disseminam (saída) os dados e informações e fornecem um mecanismo de feedback". É importante ressaltar que nenhum sistema de informação funciona sozinho. Necessitam de interferência humana, para configuração e posterior processamento das informações.

Podemos dizer então que, sistema de informação é todo recurso utilizado para prover informações e processamentos de dados destinados para qualquer que seja o uso feito dessa informação, com a finalidade de atender a organização.

Um sistema de informação possui vários elementos interrelacionados que coletam entradas, manipulam e armazenam processos, disseminam os dados e informações saídas, promovendo um resultado, destinados a tomada de decisões, com objetivo de coordenação e controle de uma organização. Porém, filtrar todo esse conjunto de dados, separando apenas aquilo que é relevante, transformando-o em informações de qualidade, pode definir o sucesso da organização.

Para [3] "um sistema de informação pode ser definido como um conjunto de componentes inter-relacionados trabalhando juntos para coletar, recuperar, processar, armazenar e distribuir informações, com a finalidade de facilitar o planejamento, o controle, a coordenação, a análise e o processo decisório em organizações".

De acordo com [4] "o sistema de informação coleta, processa, armazena analisa e dissemina informações com um determinado objetivo dentro de um contexto e como qualquer outro sistema inclui inputs (dados, instruções) e outputs (relatórios, cálculos). O sistema opera dentro de um ambiente, não necessariamente computadorizado, mesmo que atualmente a maioria seja, processa os inputs, que são enviados para os usuários e outros sistemas".

Desta forma, um sistema de informação pode ser entendido como um sistema de capacitação para a tomada de decisão, com o foco na melhoria do desempenho de uma organização.

\section{METODOLOGIA APLICADA}

A pesquisa será na abordagem bibliográfica, onde serão levantados materiais já disponíveis, em meios impressos ou digitais, para o levantamento de referências teóricas, com a finalidade de colher informações ou conhecimentos prévios, para análise das diversas posições acerca de Sistemas de Informação.

\section{II.1 CONCEITOS DE SISTEMA DE INFORMAÇÃO}

De acordo com [5], diversos setores da economia têm feito investimentos significativos em Sistemas de Informação, apontando a concorrência como o principal fator que os levam a decidir por estes investimentos.

A avaliação dos benefícios relacionados aos investimentos realizados em Sistemas de Informação, tem se tornado um componente significativo em quase tudo o que as empresas fazem [6].

Espera-se que após uma boa implantação de um [7]resultados positivos para a organização e ainda otimização da força de trabalho. Depois de implantadas os Sistemas de Informação, os gestores são responsáveis pelo uso adequado e otimizado de tais recursos, buscando a eficiência na sua utilização. Perez [8] chama atenção para a importância da gestão neste processo de implantação de novas tecnologias de informação, já que os investimentos são altos e precisam ser revertidos em benefícios para a organização.

Com a implantação de novos Sistemas de Informação, espera-se que sejam obtidas novos indicadores operacionais [9]. A utilização de indicadores concretos, ao invés de subjetivos é estimulada por [10]. Estes autores exemplificam como os Sistemas de Informação podem ajudar na melhoria das condições de trabalho, através da automação de processos monótonos.

\section{II.2 DADOS, INFORMAÇÃO E CONHECIMENTO}

Para [11] dados são registros ou fatos em estado bruto, isto é, não foram trabalhados ainda. Já para [12], o dado é visto como um elemento puro, quantificável e que, sozinho, não proporciona o entendimento de uma situação. Os dados, de forma geral, são gerados no dia-a-dia das organizações, podendo ser armazenados de forma diversa, que propicie a sua recuperação e tratamento, sempre que necessário.

Para exemplificar dados, podemos utilizar venda diária de um determinado item, valor do faturamento em $\mathrm{R} \$$, quantidade de peças de determinado produto [12]. Para [13] dados são fatos não trabalhados como nomes de pessoas, horas da jornada de trabalho, número de peças do estoque. Segundo [14], a definição de dado é uma sequência de símbolos quantificados ou quantificáveis.

Para exemplificar os diversos tipos de dados existentes, conforme mostra o Quadro 1.

Quadro 1: Tipos de Dados.

\begin{tabular}{l|l}
\hline Tipos de Dados & Representados por \\
\hline Dados Alfanuméricos & Números, letras e outros caracteres \\
\hline Dados de Imagem & Imagens, gráficos ou fotos \\
\hline Dados de Áudio & Som, ruídos ou tons \\
\hline Dados de Vídeo & Imagens em movimento ou fotos \\
\hline
\end{tabular}

Fonte: [2].

Com relação a informação, [15] destaca que é um recurso vital para as organizações, podendo assumir o papel fundamental de apoiar as estratégicas e os processos de tomada de decisão, além de proporcionar o controle das operações. Para [11], a informação é o resultado da transformação de dados organizados de forma lógica e coerente. Para [16], entendem a informação como sendo a coleta de dados, organizados e orientados.

Segundo [17], informação é o resultado dos dados existentes, devidamente tratados, a respeito de alguém ou de alguma coisa, que permita aumentar a consistência e o conteúdo dos dados coletados e selecionados.

Rezende [12] conceitua informação como sendo o resultado de dados processados, interpretados e analisados. Como exemplo de informação, podemos observar o comparativo entre as vendas de um mês do ano, com o mesmo mês do ano anterior, identificando a partir daí se a empresa obteve crescimento em suas vendas. 
Segundo [7], conhecimento é uma informação valiosa e difícil de gerenciar. É valiosa, porque foi interpretada e contextualizada por alguém. Uma pessoa refletiu sobre seu conteúdo e acrescentou sua própria interpretação, sua sabedoria, fazendo com que se tornasse ampla e difícil de gerenciar. Para Davenport [7] conhecimento é como "uma mistura fluída de experiência condensada, valores, informação contextual e insigth experimentado, a qual proporciona uma estrutura para a avaliação e incorporação de novas experiências e informações"

Rezende [12] entende que conhecimento está relacionado à habilidade de criação de um modelo que descreva o objeto desejado e indique as ações a serem implementadas.

De acordo com [11], da mesma forma que a informação é produzida a partir de dados, o conhecimento é produzido a partir de informações, quando a esta informação são agregados outros elementos.

A informação e o conhecimento são ativos cada vez mais valiosos nas organizações, podendo ser mais valioso que grandes indústrias ou ainda contas bancárias vultuosas. Cada vez mais, as empresas mais bem-sucedidas, são aquelas que utilizam melhor as informações que estão à sua disposição.

Para [14] afirma que o conhecimento não pode ser descrito, o que pode ser descrito é a informação. Igualmente, o conhecimento não depende apenas de uma interpretação pessoal e sim da experiência que o indivíduo possui sobre aquela informação. Desta forma, o conhecimento está no âmbito subjetivo do homem.

Na década de 80, o grande desafio das organizações foi a migração dos dados para informações, através da utilização de sistemas de informação. Já na década de 90, o grande desafio foi criar sistemas de informação capazes de representar e processar as informações, transformando-as em conhecimento, como resposta às necessidades dos indivíduos, grupos e cultura [12]. A figura 2 apresenta a estrutura dos dados, informação e conhecimento.

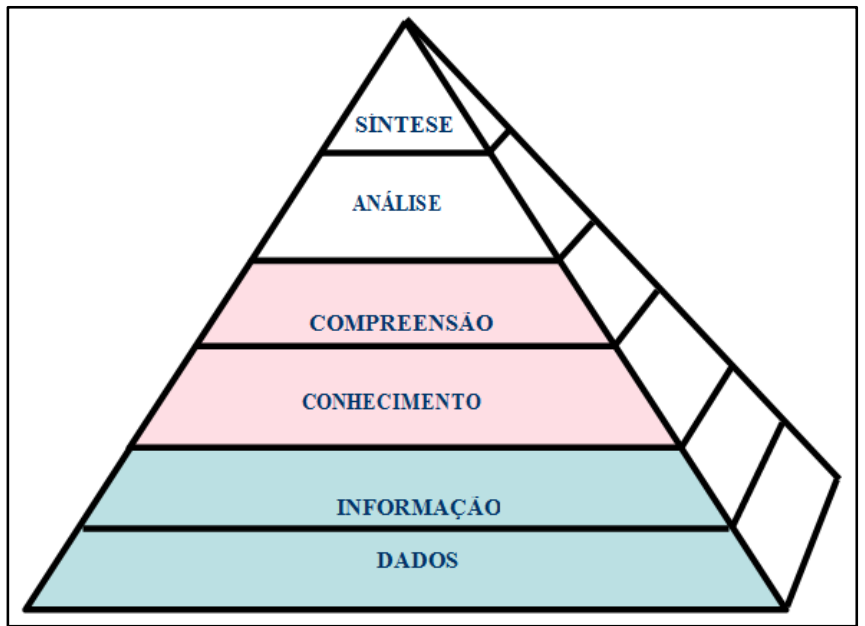

Figura 1: Dado, Informação e Conhecimento.

Fonte: Adaptado de Resende [12].

A informação, sem dúvida, é um dos ativos mais valiosos de uma organização.

Para [18], define que um sistema de informação deve ser capaz de coletar, processar, armazenar, analisar e disseminar informações para alcançar um objetivo específico. Segundo [6], um sistema de informação inclui entradas, composta por dados e instruções, saídas, compostas por relatórios e cálculos, pessoas, procedimentos e facilidades físicas.
Apesar de um sistema de informação não ter necessariamente que utilizar um computador, grande parte dos sistemas de informação das organizações modernas são informatizados, de forma a facilitar o controle de suas operações [18]. A Figura 2 apresenta uma representação gráfica de um sistema de informação.

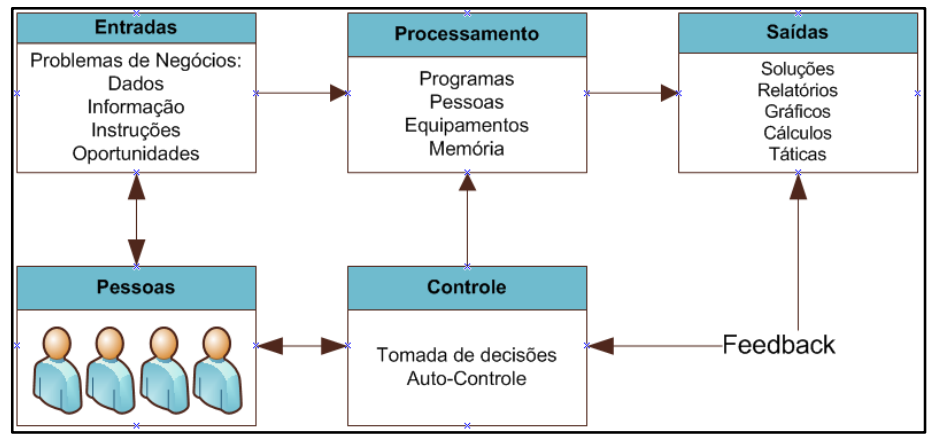

Figura 2: Representação gráfica de um sistema de informação. Fonte: Adaptado de [18].

Um sistema de informação, segundo [18] é composto por 6 elementos (Quadro 2), deixando claro que nem todos os sistemas de informação possuem esses componentes.

Quadro 2: Os 6 componentes de um Sistema de Informação.

\begin{tabular}{l|l}
\hline Componente & Descrição \\
\hline Hardware & $\begin{array}{l}\text { Conjunto de dispositivos como } \\
\text { processador, monitor, teclado e } \\
\text { impressora. Juntos, eles recebem dados e } \\
\text { informação, processa-os e apresenta-os. }\end{array}$ \\
\hline Software & $\begin{array}{l}\text { Conjunto de programas que instruem o } \\
\text { hardware como processar os dados. }\end{array}$ \\
\hline Banco de Dados & $\begin{array}{l}\text { Coleção de arquivos relacionados, tabelas, } \\
\text { relações, os quais armazenam dados e as } \\
\text { associações entre eles. }\end{array}$ \\
\hline Rede & $\begin{array}{l}\text { Sistema de conexão que permite o } \\
\text { compartilhamento de recursos entre os } \\
\text { diferentes computadores. Pode ser uma } \\
\text { rede sem fio. }\end{array}$ \\
\hline Procedimentos & $\begin{array}{l}\text { Conjunto de instruções sobre como } \\
\text { combinar os componentes acima, com o } \\
\text { propósito de processar as informações e } \\
\text { gerar a saída desejada. }\end{array}$ \\
\hline Pessoas & $\begin{array}{l}\text { Indivíduos que trabalham com o sistema, } \\
\text { comunicam-se com ele ou usam suas } \\
\text { saídas. }\end{array}$ \\
\hline
\end{tabular}

Fonte: Adaptado de [18].

Um modelo de estrutura conceitual básica de sistemas de informação é apresentada por [6] (figura 2.5) como forma de representar os 5 principais componentes e atividades de um sistema de informação:

- Pessoas (usuários finais e especialistas): são usuários finais e especialistas operadores de hardware e software;

- Hardware: equipamentos e periféricos que aceitam entrada de dados e processam informações permitindo sua visualização, como impressora, processadores, monitores, teclados, dispositivos de leitura externo, etc;

- Software: conjunto de componentes lógicos, programas, 
procedimentos, rotinas, que permitem que o hardware processe dados, como softwares, aplicativos e outros;

- Dados: um conjunto de arquivos, tabelas e outros dados relacionados entre si que armazenam dados de pessoas, lugares, coisas;

- Redes: estrutura que permite a ligação de um sistema e o compartilhamento de recursos entre diversos equipamentos.

Segundo O’Brien [6] um sistema de informação (figura 3) tem a finalidade de executar atividades de entrada e seu processamento, de forma a converter dados em informação.

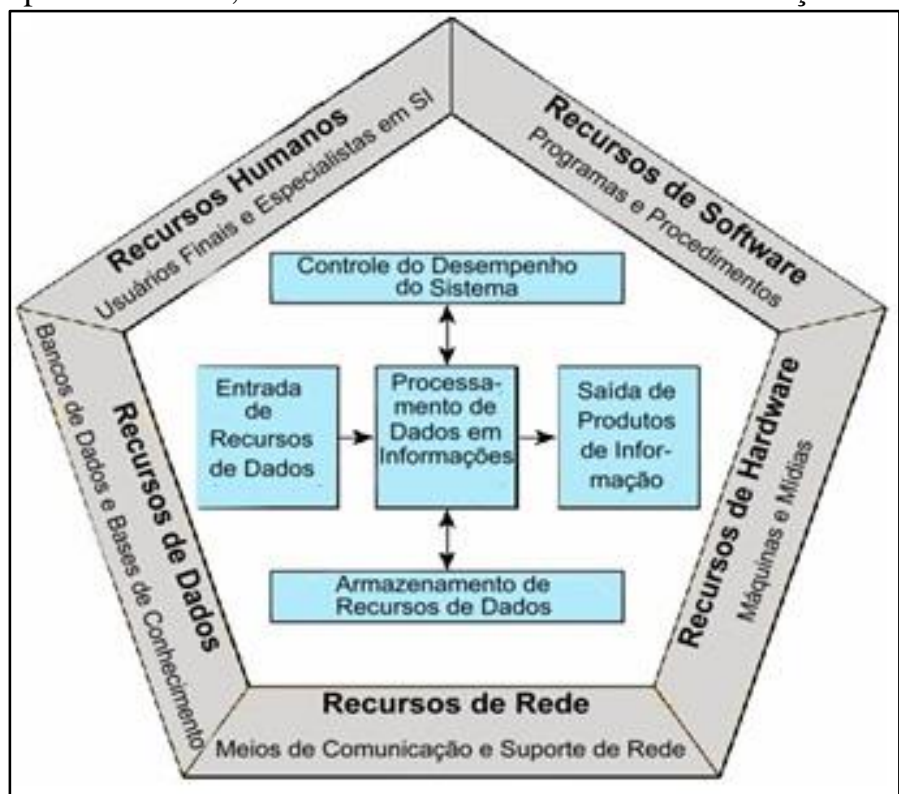

Figura 3: Os componentes de um sistema de informação. Fonte: [6].

\section{VANTAGENS E BENEFÍCIOS DOS SISTEMAS DE INFORMAÇÃO}

Compreender e apresentar de forma sintetizada as vantagens da aplicação dos sistemas de informação nas organizações, é uma necessidade cada vez maior dos gestores, tendo em vista que a utilização de tecnologia deve ser compreendida como uma das ferramentas necessárias para se alcançar maiores níveis de produtividade, gerar qualidade, competitividade e redução de custos entre outros.

Buscar a satisfação dos clientes através do atendimento às suas necessidades é necessário para garantir o crescimento das organizações. Neste sentido, muito tem se falado com relação a utilização de sistemas de informação, como forma de se antecipar às necessidades dos clientes e ainda tornar mais rápido o atendimento às suas solicitações.

Diversos benefícios obtidos pelos sistemas de informação são intangíveis, portanto, difíceis de mensuração. $\mathrm{O}$ alerta de [10] é para o fato de que é difícil avaliar a decisão de investimentos em sistemas de informação ou em projetos específicos. Alguns autores apresentam resultados obtidos com suas experiências pessoais, que demonstram os benefícios relacionados a esta tecnologia. Para [6], a estratégia competitiva das organizações pode ser obtida pelo uso de tecnologia de sistemas de informação como por exemplo:

- Redução de custos em processos operacionais;
- Diferenciação em produtos e serviços;

- Crescimento organizacional.

Uma forma de se avaliar os resultados da implantação de sistemas de informação é a avaliação de indicadores de desempenho, com medidas objetivas, que possam ser comparadas com o resultado histórico da organização ou ainda com o resultado de outras organizações [10]. Como sugestão, ou autores indicam a adoção de indicadores de custo médio do setor, com valores gastos pelas empresas congêneres, de forma a avaliar o investimento das empresas neste segmento.

Duas possibilidades de benchmark são relacionadas por [10]: o de números que oferece medidas numéricas para se avaliar o desempenho e o de melhores práticas, que oferece a forma de se executar as melhores práticas do mercado. No Quadro 3 pode-se observar uma ilustração com relação aos tipos de benchmark:

Quadro 3: Tipos de Benchmarks.

\begin{tabular}{l|ll}
\hline Benchmark de Números & $\begin{array}{l}\text { Benchmark de } \\
\text { Práticas }\end{array}$ \\
\hline $\begin{array}{l}\text { Gastos com Sistema de } \\
\text { Informação em percentual da } \\
\text { receita total. }\end{array}$ & $\begin{array}{l}\text { Obtenção de } \\
\text { práticas na área. }\end{array}$ \\
\hline $\begin{array}{l}\text { Percentual de "tempo de } \\
\text { espera" } \begin{array}{l}\text { (quando } \\
\text { computador nãos } \\
\text { disponível). nacsá }\end{array}\end{array}$ & $\begin{array}{l}\text { Comparação com empresas } \\
\text { do setor. }\end{array}$ \\
\hline $\begin{array}{l}\text { Uso da CPU (em percentual } \\
\text { da capacidade total). }\end{array}$ & $\begin{array}{l}\text { Comparação com empresas } \\
\text { de outros setores. }\end{array}$ \\
\hline $\begin{array}{l}\text { Percentual dos projetos de } \\
\text { Sistema de Informação } \\
\text { concluídos dentro do prazo e } \\
\text { orçamento. }\end{array}$ & $\begin{array}{l}\text { Comparação com outras } \\
\text { unidades internas que usam } \\
\text { Sistemas de Informação de } \\
\text { forma mais eficiente. }\end{array}$ \\
\hline
\end{tabular}
Fonte: [10].

Diversos fatores levam as empresas a investir em Tecnologia da Informação, principalmente em sistemas de informação, pois um sistema de informação bem disseminado, pode consolidar a organização, principalmente no que diz respeito a qualidade, produtividade e participação [19].

Os autores [20] relacionam os objetivos principais que levam uma organização a implantar sistemas de informação:

- Excelência operacional (produtividade, eficiência e agilidade);

- Desenvolvimento de novos produtos e serviços;

- Estreitar o relacionamento com o cliente;

- Agilizar a tomada de decisão;

- Promover a vantagem competitiva;

- Assegurar a sobrevivência no mercado.

Para [20], as organizações que conseguirem através da implantação de um sistemas de informação, a excelência operacional, assim como a colocação de novos produtos no mercado, certamente obterá vantagem competitiva e, consequentemente, estará à frente dos seus concorrentes.

Os autores [20] afirmam também que os sistemas de informação são cada vez mais imprescindíveis para a prática diária dos processos de negócios, sendo necessário a sua utilização para que se tornem competitivas, organizadas e aptas a responder às mudanças requeridas pelo mercado. 
Segundo [21], alguns dos benefícios e vantagens dos sistemas de informação são:

- Redução dos custos das operações;

- Melhoria no acesso às informações, proporcionando relatórios mais precisos e rápidos;

- Aumento da produtividade;

- Melhoria na tomada de decisões;

- Melhoria na estrutura organizacional;

- Melhoria na estrutura de poder, propiciando maior poder para aqueles que entendem e controlam o sistema;

- Otimização na prestação de serviços aos clientes;

- Melhor interação com fornecedores;

- Melhoria das atitudes e atividades dos colaboradores;

- Redução da mão de obra burocrática,

Para [22] a empresa que detiver, organizar, dominar e valorizar a informação e o conhecimento do meio ambiente (interno e externo) em que estiver inserida, terá maior vantagem competitiva nos negócios e maior possibilidade de permanecer no mercado.

Segundo [23], com a implantação de sistemas de informação, a empresa conquista vantagem competitiva e pode focar em nichos específicos de mercado, conseguir liderança em custos e diferenciação de produtos.

Os sistemas de informação são um instrumento que interliga todos os setores da empresa. Organizações que não trabalham com sistemas de informação podem ter informações falhas, ou de difícil acesso, prejudicando a tomada de decisão e até incorrendo em erros considerados graves, como por exemplo o envio de informações incorretas para órgãos federais, municipais e estaduais.

Os sistemas de informação precisam ser simples e fáceis de utilizar. Organizações que trabalham com sistemas complexos ou que não estejam devidamente customizados para a sua realidade, podem sofrer diariamente com erros em processos rotineiros, que findam se tornando difíceis de correção e levando a tomada de decisões incorretas.

Para [23] é comum os gestores trabalharem sem ter informações precisas, na hora que precisam, fazendo com que precisem tomar decisões baseadas em seu conhecimento sobre o assunto, previsões ou contando com a sorte. Esta ação proporciona problemas na produção, que pode ser excessiva ou insuficiente.

Os sistemas de informação precisam ser desenvolvidos ou customizados de acordo com as necessidades da organização. As manutenções e atualizações, também precisam levar em conta a real necessidade da organização e do mercado, de forma que o sistema se mantenha simples e fácil de manusear pelos colaboradores.

A manutenção corretiva dos sistemas de informação necessita ser precisa, de forma a eliminar os gargalos existem pelos problemas detectados, evitando que as informações fiquem imprecisas e que novos erros voltem a ocorrer.

Com a globalização, os sistemas de informação cresceram muito. Segundo [23], o principal motivador para as organizações investirem e aprimorarem seus sistemas de informação é a intenção delas de atingir seis importantes objetivos organizacionais: excelência nos processos, lançamento de novos produtos, prestação de serviços e modelos de negócios, relacionamento mais próximo com clientes e fornecedores, maior vantagem competitiva e sobrevivência no mercado.

\section{IV.CONSIDERAÇÕES FINAIS}

Com a realização do presente estudo, pôde-se constatar que os sistemas de informação são fornecedores de informações estratégicas que podem alavancar os negócios de uma organização.

É a interação dos componentes dos sistemas de informação com o ser humano que faz com que ele tenha funcionalidade e utilidade para organização. Através dos benefícios dos sistemas de informação a empresa pode evoluir no mercado, pois através dos sistemas de informação a empresa pode ter o controle de colaboradores, dos seus números no mercado, informações dos concorrentes entre outros benefícios que são trazidos a empresa.

De um modo geral, as informações compõem um dos maiores e mais valiosos ativos da empresa, neste sentido as empresas que possuem um sistema de informação e que mantém o seu pessoal motivado a utilizar estas informações será mais dinâmica, agressiva e atuante, uma vez que as decisões tomadas serão mais acertadas.

O uso adequado do sistema de informação proporciona uma combinação estruturada de informações, recursos humanos, tecnologia de informação e práticas de trabalho organizadas de forma a permitir o melhor atendimento dos objetivos da organização.

A tecnologia estará cada vez mais presente na rotina diária das organizações e os sistemas de informação tendem a ser cada vez mais utilizados pelos gestores e pela alta direção, com o objetivo de tomar decisões estratégicas que possam melhorar a rotina da organização. Vale lembrar que da mesma forma que um novo sistema de informação pode criar oportunidades para uma organização ele pode ser responsável pelo fracasso de outras organizações, que não conseguem tirar proveito dos benefícios do sistema e, consequentemente, não conseguem se posicionar adequadamente no mercado em que atuam.

\section{AGRADECIMENTOS}

Ao sempre Mestre e grande amigo Prof. Dr. Jorge Laureano Moya Rodríguez pela sua dedicação, paciência, disponibilidade e incentivo.

\section{REFERÊNCIAS}

[1] Queiroz, G.F., A contribuição dos arquivistas nos sistemas de SGQ. Queiroz, G.F., A contribuição dos arquivistas nos sistemas de SGQ. Monografia do Curso de Pós Graduação Lao Sensu. Universidade Canbdido Mendes. Rio de Janeiro. 2011.

[2] Stair, R.M., G.W. Reynolds, and F.S.C. da Silva, Princípios de sistemas de informação: uma abordagem gerencial. 1998. [3] LAUDON, K.C., LAUDON Jane Price. Sistemas de
informação, 1999.

[4] Turban, E., et al., Tecnologia da Informação para Gestão-: Transformando os Negócios na Economia Digital. 2010: Bookman.

[5]Lunardi, G.L., J.L. Becker, and A.C.G. Maçada, Relacionamento entre investimentos em tecnologia de 
informação (TI) e desempenho organizacional: um estudo cross-crountry envolvendo os bancos brasileiros, argentinos e chilenos. Anais do ENANPAD, 2003.

[6]O’brien, J.A., Sistemas de informação. São Paulo, 2002. Davenport, T.H., Ecologia da informação: por que só a tecnologia não basta para o sucesso na era da informação. 2000: Futura.

[8]Perez, G., Avaliação e escolha de fornecedores de tecnologia da informação: estudos de casos múltiplos. 2003. 2003, Dissertação (Mestrado)-FEA, USP, São. Paulo.

[9]Kaplan, R.S. and D.P. Norton, A estratégia em ação: balanced scorecard. 1997: Gulf Professional Publishing.

[10]Turban, E., J.C. Wetherbe, and E. Mclean. Tecnologia da informação para gestão. 2004: Bookman.

[11] Beal, A.Gestão estratégica da informação: como transformar a informação em fatores de crescimento e de alto desempenho nas organizações. São Paulo: Atlas, 2004.

[12] Rezende, S.O., Sistemas inteligentes: fundamentos e aplicações. 2003: Editora Manole Ltda.

[13] Stair, R.M.R., G.W.R.M. Stair, and G.W. Reynolds, Principios de sistemas de información: enfoque administrativo. 2010: Cengage Learning.

[14] Setzer, V.W. Dado, informação, conhecimento e competência. DataGramaZero Revista de Ciência da Informação, n. $0,1999$.

[15] Beuren, I., Gerenciamento da informação: um recurso no processo estratégico empresarial. Editora Atlas, 2000.

[16] McGee, J.V. and L. Prusak, Gerenciamento estratégico da informação. 1994: Elsevier Brasil.

[17]Batista, E.d.O., Sistemas de informação. São Paulo: Saraiva, 2004.

[18] Turban, E., et al., Information technology for management, (With CD). 2008: John Wiley \& Sons.

[19] Moscove, S.A., et al., Sistemas de informações contábeis. 2002: Atlas.

[20] Laudon, K.C. and J.P. Laudon, Sistemas de informação gerenciais: administrando a empresa digital. 2005: Pearson Prentice Hall.

[21] Schoupinski, A.B., et al., Sistemas de informação: um estudo sobre a utilização e vantagens dos sistemas de informação gerencial. V Encontro Paranaense de Pesquisa e Extensão em CIências Sociais e Aplicadas e VIII Seminário do Centro de Ciências Sociais Aplicadas de Casacavel. Paraná. 2009.

[22]Rezende, D.A. and A.F.d. Abreu, Tecnologia da informação aplicada a sistemas de informação empresariais. São Paulo: Atlas, 2000. 3.
[23]Keneth, C.L. and J. Laudon, Sistemas de Informações Gerenciais. 2007, São Paulo: Editora Pearson Brasil, Edição. 